\title{
Lorentz Transformation from Symmetry of Reference Principle
}

\author{
M. Dima \\ Nuclear Science \& Technology Dept., \\ Harbin Engineering University, \\ Harbin, Heilongjiang, CN-150000, China
}

\begin{abstract}
The Lorentz Transformation is traditionally derived requiring the Principle of Relativity and lightspeed universality. While the latter can be relaxed, the Principle of Relativity is seen as core to the transformation. The present letter relaxes both statements to the weaker, Symmetry of Reference Principle. Thus the resulting Lorentz transformation and its consequences (time dilatation, length contraction) are, in turn, effects of how we manage space and time.
\end{abstract}

PACS numbers: 13.25.Hw, 13.25.-k, 14.40.Nd

Starting with the 1905 paper of A. Einstein in Ann. Phys. [1] the Lorentz Transformation has been traditionally derived based on the Principle of Relativity and lightspeed universality. A number of studies [2] have shown that light-speed universality is not needed - the first such publication (1906) being owed to H. Poincaré [3]. Group theory expresses the transitivity property of relativity $(\mathrm{C}$ relative to $\mathrm{A}$, if $\mathrm{A}$ to $\mathrm{B}$ and $\mathrm{B}$ to $\mathrm{C}$ ) in the form of the group closure relation, respectively the product of two group elements being another element of the group. Pure relativity transformations however, cannot form a group on their own, needing rotations to "close" the group. As such, the full group is not immediate from the Principle of Relativity and needs to be specified (in this case a group of transformations invarying the metric: $\boldsymbol{\Lambda}^{\dagger} \mathbf{G} \boldsymbol{\Lambda}=\mathbf{G}$, where $\boldsymbol{\Lambda}$ are the transformations and $\mathbf{G}$ the metric). Entering the Lorentz group however, is equivalent to admitting light speed invariance. In this sense the Principle of Relativity and (indirectly) light speed invariance are core to the Lorentz Transformation [4].

The present letter shows however, that neither statement is necessary and that the Lorentz transformation stems from the simpler (weaker) Principle of Symmetry of reference systems. Further more, the Minkowsky metric is not unique in defining relativity. There are two possible classes of transformations, one invarying the Minkowsky and the other the Euclidian metric. The adhoc terminology of Minkowsky and Euclidian relativities will be thus adopted throughout this letter.

Consider two coordinate systems in motion that at some point were at rest relative to each other and were aligned to have the same orientation, offset and (Euclidian) space-metric. The transformation between such coordinate systems is:

$$
\left(\begin{array}{c}
d t \\
d \vec{x}
\end{array}\right)^{\prime}=\underbrace{\left(\begin{array}{ll}
\text { scalar }_{1 \times 1} & \text { vector }_{1 \times 3} \\
\text { vector }_{3 \times 1} & \text { tensor }_{3 \times 3}
\end{array}\right)}_{\Lambda}\left(\begin{array}{c}
d t \\
d \vec{x}
\end{array}\right)
$$

where the dimensions of the objects involved is given by the subscripts. In general the transformation should be an integral, non-linear transformation, however general considerations about space-time limit the range of possible transformations to constant linear transformations:

1. locality - implies that the transformation must be point-to-point;
2. homogeneity - implies that the transformation must not depend on the relocation of the coordinate system, hence linear;

3. isotropy - implies that the mathematical objects in the transformation cannot be "pseudo"-objects, since coordinate system space/time inversion must not affect the transformation. Also, the vectors must be parallel to $\vec{v}$ - the relative velocity between the systems in causa, as no new direction in space can be introduced (isotropy). Likewise, the general form of the tensor - less a $(\times \vec{v})$ pseudo-tensorial part ruled out by isotropy, is $\lambda \mathbf{C}_{\|}+\mu \mathbf{C}_{\perp}$, where $\mathbf{C}_{\|}$selects vector components parallel to $\vec{v}$, and $\mathbf{C}_{\perp}$ components perpendicular to $\vec{v}$. The scalars must be functions of $|\vec{v}|$, as there exists no preferred direction in space.

The transformation can be thus written as:

$$
\boldsymbol{\Lambda}=\gamma_{v}\left(\begin{array}{cc}
1 & -\vec{v} / c_{v}^{2} s \\
-\psi_{v} \vec{v} & \lambda_{v} \mathbf{C}_{\|}+\mu_{v} \mathbf{C}_{\perp}
\end{array}\right)
$$

with the scalars fulfilling the roles described above and $s$ a sign factor $s= \pm 1$. For reasons evident later the two shall be termed Minkowsky $(s=+1)$ and Euclidian relativity $(s=-1)$.

The apparent-velocity of an object moving with $\vec{u}$ in the base-system is seen in the moving-system as:

$$
\vec{u} \ominus \vec{v}=\frac{-\psi_{v} \vec{v}+\lambda_{v} \vec{u}_{\|}+\mu_{v} \vec{u}_{\perp}}{1-\vec{v} \vec{u} / c_{v}^{2} s}
$$

the scalar $c_{v}$ having units of speed.

The following are evident:

1. for $\vec{v} \rightarrow 0$ the transformation is unitary:

$$
\lim _{v \rightarrow 0} \Lambda_{\vec{v}}=\mathbf{1}
$$

$$
\text { hence } \gamma_{v}, \lambda_{v}, \mu_{v}=1 \text {, }
$$

2. $\vec{v} \ominus \overrightarrow{0}=-\overrightarrow{0} \ominus \vec{v}$ thus $\psi_{v}=1$.

3. $\vec{v} \ominus \vec{v}=\overrightarrow{0} \quad$ thus $\quad \lambda_{v}=\psi_{v}=1$.

4. $\boldsymbol{\Lambda}_{-\vec{v}}=\boldsymbol{\Lambda}_{\vec{v}}^{-1} \quad$ thus:

- $\gamma_{v}= \pm 1 / \sqrt{1-\vec{v}^{2} / c^{2} s}$, the valid sign (+) being determined from $\lim _{v \rightarrow 0}$, 
- $\mu_{v}= \pm 1 / \gamma_{v}$, the valid sign $(+)$ being determined from $\lim _{v \rightarrow 0}$.

5. $\left|\vec{v}_{2} \ominus \vec{v}_{1}\right|=\left|\vec{v}_{1} \ominus \vec{v}_{2}\right|$ implies $c_{1}=c_{2}=c$ a constant and $s_{1}=s_{2}=$ the same sign.

From the apparent-velocity law, the combined speed is:

$$
|\vec{u} \ominus \vec{v}|^{2}=\frac{(\vec{u}-\vec{v})^{2}-s(\vec{u} \times \vec{v})^{2}}{\left(1-\vec{u} \vec{v} / c^{2} s\right)^{2}}
$$

confirming $v_{\text {lim }} \leq c$ for $s=+1$ at point (4) above. The two cases are somewhat similar for $v$ or $u>c$, but differ significantly for $v$ and $u<c$.

The transformation is thus now:

$$
\boldsymbol{\Lambda}=\left(\begin{array}{cc}
\gamma_{v} & -\gamma_{v} \vec{v} / c^{2} s \\
-\gamma_{v} \vec{v} & \gamma_{v} \mathbf{C}_{\|}+\mathbf{C}_{\perp}
\end{array}\right)
$$

respectively the well known Lorentz transformation for $s=+1$. The meaning of $c$ is related to causality: for both Minkowsky and Euclidian relativities the transformed time interval versus proper time is $d t^{\prime}=\gamma_{v}\left(1-\vec{v} \vec{u} / c^{2} s\right) d \tau$, respectively a causal transformation for $v<c$. For $c \rightarrow \infty$ the Galilean transformation is recovered.

The Principle of Relativity (group "closure" - modulo a rotation) has not been used thus far:

$$
\boldsymbol{\Lambda}_{1} \boldsymbol{\Lambda}_{2}^{-1}=\mathbf{R} \boldsymbol{\Lambda}_{12}
$$

where $\boldsymbol{\Lambda}_{1,2}$ are two coordinate transformations, $\boldsymbol{\Lambda}_{12}$ the system- 1 to system- 2 transformation and $\mathbf{R}$ an alignment rotation $\left(\vec{v}_{2} \ominus \vec{v}_{1}\right)=-\mathbf{R}\left(\vec{v}_{1} \ominus \vec{v}_{2}\right)$ that appears due to boost when referencing is done via alignment with a (third party) base-system. In a strong sense the Principle of Relativity is the group "closure" [3] relation (7) satisfied by both Euclidian and Minkowsky relativities, however in a weaker form it has been used in relations (2), (3) and (5) as the Symmetry of Reference Principle. The only isotropic metrics invariant under the transformations are the Euclidian $(s=-1)$ and the Minkowsky $(s=+1)$ metric.

Principially tachyons are allowed in both Minkowsky and Euclidian relativities, with the notable difference that continuous acceleration across the light-cone is possible only in the latter. For tachyons time can be seen as running backward if $\vec{u} \vec{v} s>c^{2}$. Since the beginning and end points of a tachyon track are not labeled, the tachyon could look like a sub-luminous particle. In certain contexts the apparent speed of a tachyon can even be $v_{\text {tachyon }} \ll c$. Another interesting aspect of tachyons is their apparent charge $\rho^{\prime}=\gamma_{v} \rho\left(1-\vec{v} \vec{u} / c^{2} s\right)$, of opposite sign to $\rho$ when the apparent time of the tachyon is running backward. Apparent time-reversal couples well with charge-conjugation, which is supposed to turn all currents into anti-currents, rather than each one individually - with the $\gamma_{\text {Dirac }}$ matrices of its own quantum space. With respect to other charge-conjugation representations, time-reversal $\left(\gamma^{0} \gamma^{5}\right.$ in the Dirac theory) is
Dirac-representation independent and space-coordinate independent. Minkowsky relativity has the problem of imaginary $\gamma_{v}=i / \sqrt{v^{2} / c^{2}-1}$ for $v>c$, however tachyon dynamics is expressed in both Minkowsky and Euclidian relativity with $\gamma^{2}$ :

$$
m_{0} \vec{a}=\left(\frac{1}{\gamma^{2}} \mathbf{C}_{\|}+\mathbf{C}_{\perp}\right) \frac{d \vec{p}}{d t}
$$

the acceleration parallel to the force diverging with increasing speed. In Minkowsky relativity however, as particles approach the light-cone (from above, or below) acceleration in the direction of the force is annulled.

I am thankful for the hospitality during completion of this work to the Nuclear Science \& Technology Dept. of the Harbin Engineering University - China under a Foreign Expert grant.

[1] A. Einstein, Ann. Phys. 17, 891 (1905).

[2] Examples in J.M. Levy-Leblond, Am. J. Phys. 44, 271 (1976).

[3] H. Poincaré, Rend. Circ. Mat. Palermo, 21, 129 (1906).

[4] V. Berzi, V. Gorini, J. Math. Phys. 44, 271 (1976).

[5] M. Dima, JETP Lett. 72, 541 (2000). 